\title{
On the Design of Underwater Acoustic Cellular Systems
}

\author{
Milica Stojanovic \\ Massachusetts Institute of Technology \\ millitsa@mit.edu
}

\begin{abstract}
The design of a cellular underwater network is addressed from the viewpoint of determining the cell size and the frequency reuse pattern needed to support a desired number of users operating over a given area within a given system bandwidth. By taking into account the basic laws of underwater acoustic propagation, it is shown that unlike in the terrestrial radio systems, both the cell radius $R$ and the frequency reuse number $N$ must satisfy a set of constraints in order to constitute an admissible solution (which sometimes may not exist). The region of admissible $(R, N)$ defines the possible network topologies. It is determined by the user density and the system bandwidth $(\rho, B)$, and by the required signal-to-interference ratio and per-user bandwidth $\left(\mathbf{S I R}_{0}, W_{0}\right)$. The range of admissible solutions also depends on the choice of operating frequency region. Moving to a higher frequency region than that dictated by SNR maximization, improves the SIR and yields a greater system capacity.
\end{abstract}

\section{INTRODUCTION}

Cellular networks have revolutionized terrestrial mobile communications by introducing the concept of spatial frequency reuse [1]. The simple idea of using the same frequency band in two cells that are sufficiently far apart so that they do not cause interference to each other enables spatial reuse of bandwidth, which in turn ensures coverage of a large area within the practical constraints of finite bandwidth. Since bandwidth is at premium in an acoustic channel, the concept of frequency reuse is an appealing one, and, given the immense success and practicality of terrestrial cellular systems, the question naturally arises as to how does the cellular concept apply to an underwater acoustic environment.

Motivated by this question, we address the top-level system design and the implications that acoustic propagation bears on the frequency reuse concept. As an example application, one may think of a fleet of autonomous vehicles, deployed on a collaborative mission to map a wide area of the ocean floor. The base stations in such a system may be mounted on the surface buoys (radio-based infrastructure) or on the bottom (cable-based infrastructure). One could also use both surface and bottom stations to provide full volume coverage in an underwater network. For simplicity, we restrict our attention to a two-dimensional problem.

The first task in the design of a cellular network is the selection of network topology, i.e. determination of the cell size (radius) and the reuse pattern. In other words, the practical questions that one wants to answer are the following: For a given distribution of users and the desired information throughput, what should the coverage area of one base station be? How far does another base station operating in the same frequency band has to be? According to what pattern should the frequency bands be reused?

In a terrestrial radio environment, this problem has a very simple solution that leads to the well-known reuse pattern of 7 , and selection of the smallest practical cell radius [1]. In an underwater acoustic environment, however, the solution is complicated by the fact that the path loss does not consist only of the spreading loss (which grows with distance as $d^{k}$, where $k$ is usually a number between 1 and 2), but the absorption loss as well (which grows with distance as $a^{d}$, and depends on the frequency through the factor $a$ ). We show that due to this fundamental difference, simple principles of cellular radio system design do not apply to an underwater acoustic environment. Instead, complex relationships between the system parameters are involved, which lead to a set of constrained solutions, and, sometimes, to a situation in which it is not possible to use the cellular concept at all.

the problem of underwater cellular system design is formulated in Sec.II. This section also outlines the admissible solutions, and discusses the results through a series of numerical examples. Conclusions are summarized in Sec.III.

\section{SySTEM DESIGN}

We consider a system layout that follows the basic cellular concept of spatial frequency reuse that comes with timedivision or frequency-division multiple-access [1]. Specifically, we address the following scenario: given a density of users $\rho$ [users $\left./ \mathrm{km}^{2}\right]$ and a total system bandwidth $B[\mathrm{~Hz}]$, we want to find the cell radius $R[\mathrm{~m}]$ and the frequency reuse number $N$, such that the number of users per $\mathrm{Hz}$ of occupied bandwidth is maximized, subject to the fact that the following conditions are met:

(i) the co-channel signal to interference ratio remains greater than a pre-determined level, $\mathrm{SIR} \geq \mathrm{SIR}_{0}$, and

(ii) the bandwidth (throughput) per user remains greater than a pre-specified value, $W \geq W_{0}$.

\section{A. Signal to interference ratio}

We start by evaluating the co-channel SIR, taking into account the acoustic propagation loss. Assuming a hexagonal cell geometry, the co-channel interference has as its dominant component the signals coming from the nearest six co-channel cells. The least favorable conditions occur at the cell edge, 
where the SIR is approximately defined by the ratio of powers

$$
\mathrm{SIR}=\frac{P(R)}{6 P(D)}
$$

Here, $R$ is the distance traveled by the desired signal, and $D$ is the distance traveled by the interfering signal. In a twodimensional system geometry, the two distances are related by $D=Q R$, where $Q=\sqrt{3 N}$, and the reuse number is given in the form $N=i^{2}+i j+j^{2}$, with $i, j$ integers [1].

The acoustic path loss, experienced by a signal of frequency $f$ traveling over a distance $R$ is given by

$$
A(R, f)=A_{0} R^{k} a^{R}(f)
$$

where $A_{0}$ is a normalizing constant, $k$ is the spreading factor, and $a(f)$ is the absorption coefficient. Without the loss of generality, we will assume in the later numerical examples a practical spreading factor $k=1.5$, and absorption according to Thorp, which is reproduced in Fig.1.

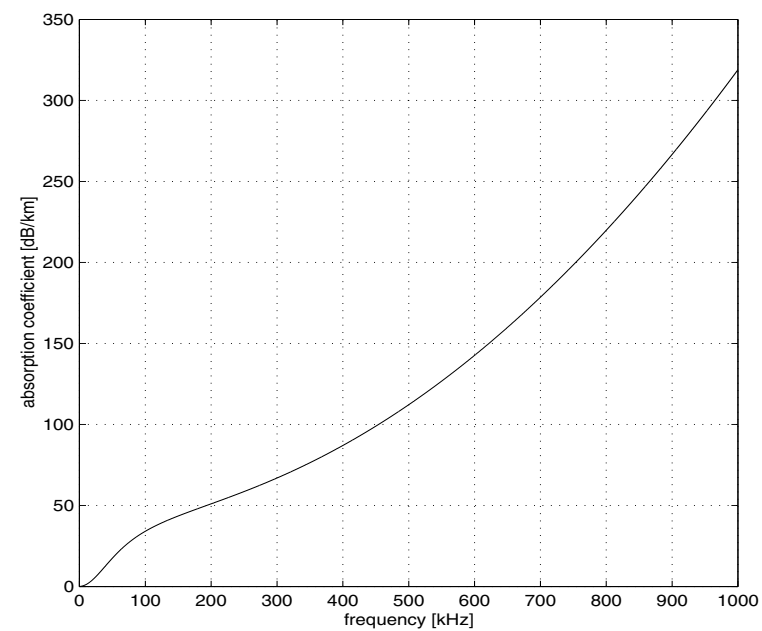

Fig. 1. Absorption coefficient $a(f)$ is given in $\mathrm{dB} / \mathrm{km}$ as $10 \log a(f)=$ $0.11 f^{2} /\left(1+f^{2}\right)+44 f^{2} /(4100+f)+2.75 \cdot 10^{-4} f^{2}+0.003$, where $f$ is the signal frequency in $\mathrm{kHz}$. This empirical formula is valid for frequencies greater than a few hundred $\mathrm{Hz}$ [2].

The signal power at a distance $R$ from the transmitter is now evaluated as

$$
P(R)=S \int_{f_{\min }}^{f_{\min }+B_{0}} A^{-1}(R, f) d f
$$

where $S$ is the power spectral density of the transmitted signal (assumed to be constant and as such not relevant for the SIR), and the integration is carried over the frequency band occupied by the signal, starting at some $f_{\min }$ and extending over a bandwidth $B_{0}$.

The signal bandwidth $B_{0}$ depends on the multiple-access technique used. If TDMA is used, it equals the bandwidth allocated to one cell, $B_{0}=B / N$. If FDMA is used, it equals the width of a frequency channel allocated to one of $U$ users sharing a cell, $B_{0}=(B / N) / U$. In what follows, we shall assume a TDMA system, keeping in mind that the analysis can easily be modified to accommodate FDMA. (Note that in an FDMA system different channels experience different attenuation due to the frequency-dependence of the acoustic path loss. In particular, higher-frequency channels experience greater attenuation. However, this is true both for the desired signal and for the interfering signals, with the overall effect that the SIR improves with an increase in frequency. Hence, to ensure that the worst-case conditions are met, the system design should be carried out for the lowest frequency channel, which is the one at $f_{\min }$.)

Using the acoustic path loss to determine the received powers, the SIR condition can be expressed in terms of the cell radius $R$ and the reuse factor $Q$ as

$$
\mathrm{SIR}=\frac{1}{6} Q^{k} \frac{I(R)}{I(Q R)} \geq \operatorname{SIR}_{0}
$$

where

$$
I(x)=\int_{f_{\text {min }}}^{f_{\min }+B_{0}} a^{-x}(f) d f
$$

At this point, we may note the difference between an acoustic and a radio system: in a radio system, the path loss does not contain the frequency-dependent absorption term, and, consequently, the SIR reduces to $Q^{k} / 6$. The SIR condition is thus rendered independent of the cell radius $R$, and the reuse number $N$ is easily determined from it. (Its typical value in cellular radio systems is $N=7$, which ensures SIR greater than $17 \mathrm{~dB}$ for the two-ray ground reflection model with path loss exponent $k=4$.) In the acoustic channel, this is not the case. The SIR depends both on the reuse number and the cell radius through the terms $I(R)$ and $I(Q R)$.

In order to obtain an explicit expression for the SIR as a function of $R$ and $N$, one may be tempted to make an approximation $a(f) \approx a\left(f_{\min }\right)=a_{0}$, which would yield

$$
I(x) \approx a_{0}^{-x}
$$

and

$$
\mathrm{SIR} \approx \frac{1}{6} Q^{k} a_{0}^{(Q-1) R}
$$

However, such an approximation is acceptable only for $B_{0}<<$ $f_{\text {min }}$, which is rarely the case in an acoustic system. Fig.2 shows the function $I(x)$ and its "approximation" (6) for an arbitrary selection of the system parameters: $f_{\min }=10 \mathrm{kHz}$ and $B_{0}=7 \mathrm{kHz}$ (the latter would correspond to a total bandwidth $B$ of about $50 \mathrm{kHz}$ and $N=7$ in a TDMA system).

In addition to being dependent on the cell radius and the reuse number, the SIR also depends on the band-edge frequency $f_{\min }$ and, to a lesser extent, on the bandwidth $B_{0}$. Fig. 3 illustrates the SIR as a function of $f_{\min }$. The SIR increases with frequency since $a(f) \geq 1 \forall f$. Using a higher frequency band thus ensures a greater SIR; however, it results in higher attenuation, making the signal more vulnerable to noise. The signal to noise ratio (SNR) is also illustrated in the figure, along with the resulting signal to noise plus interference ratio (SINR). A practical system is normally designed such that the noise is negligible with respect to interference, i.e. SNR $>>$ SIR, or, equivalently, SINR $\approx$ SIR (hence SIR is used as a figure of merit for the system performance). 


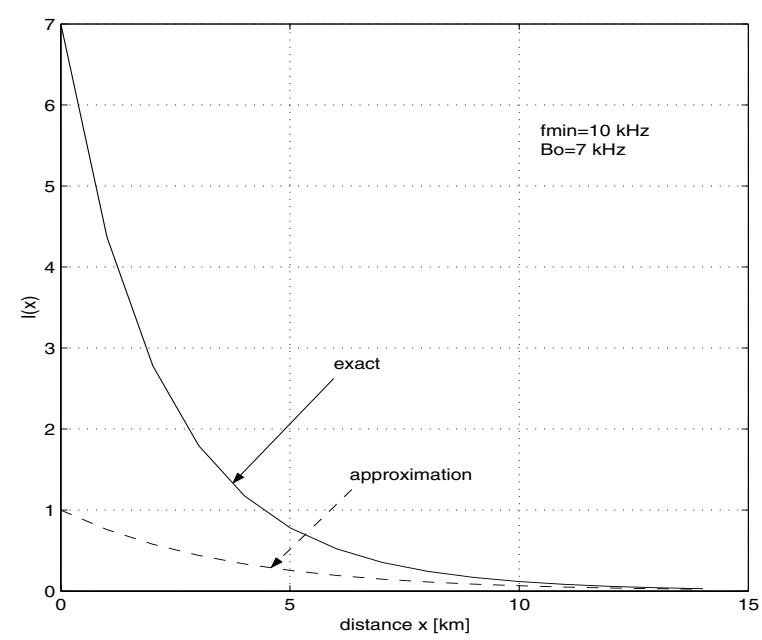

Fig. 2. $\quad I(x)$ as defined in (5). The approximation (6) is a poor one for a wideband acoustic system.

Selection of the optimal $f_{\min }$ is obviously influenced by many factors, including the physical constraints of the transducers, and the power budget. For the moment, we lay these large-scale system design issues aside, and focus on the selection of the cell radius and the reuse pattern, assuming that $f_{\min }$ is set a-priori. In the numerical examples that follow, we will use $f_{\min }=10 \mathrm{kHz}$.

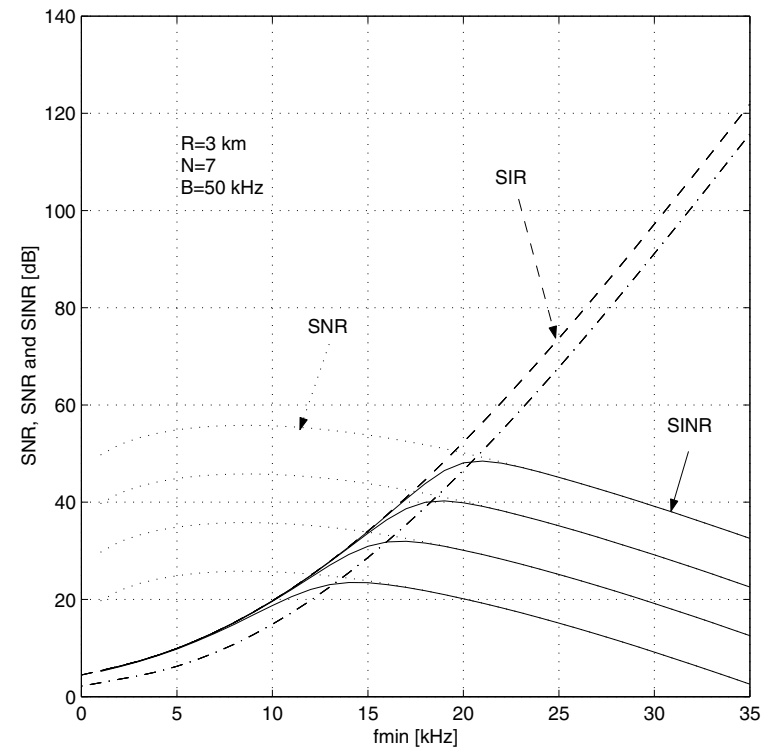

Fig. 3. Signal to noise, signal to interference, and signal to noise plus interference ratios vs. $f_{\min }$. The approximation (7) for the SIR is shown in dash-dot line below the actual SIR. Several transmission power levels are used (ranging from 120 to $150 \mathrm{~dB}$ re $\mu \mathrm{Pa}$ in steps of $10 \mathrm{~dB}$ ): changing the transmission power affects the SNR, but not the SIR. Noise power is calculated using ambient noise power spectral density corresponding to moderate shipping activity and no wind, as in [3].

\section{B. Cell radius and the reuse number}

Fig.4 shows the SIR as a function of the cell radius for different values of the reuse number. The SIR increases with both $R$ and $N$. Thus, in order for the SIR to be greater than the design value $\mathrm{SIR}_{0}$, the cell radius has to be greater than some minimum, which we express as

$$
R \geq R_{0}(N)
$$

The minimal cell radius depends on the reuse pattern $N$, and also on the required SIR and the system bandwidth. There is no closed form expression for $R_{0}(N)$, unless the approximation (6) is used, which would yield

$$
R_{0}(N) \approx \frac{10 \log \mathrm{SIR}_{0}+4-k \cdot 10 \log Q}{(Q-1) 10 \log a_{0}}
$$

Again, this approximation should be used only for illustrative purposes, to gain insight into the rate of decay of the minimal radius with $N$. The exact function $R_{0}(N)$ is shown in Fig.5, which we will discuss shortly.

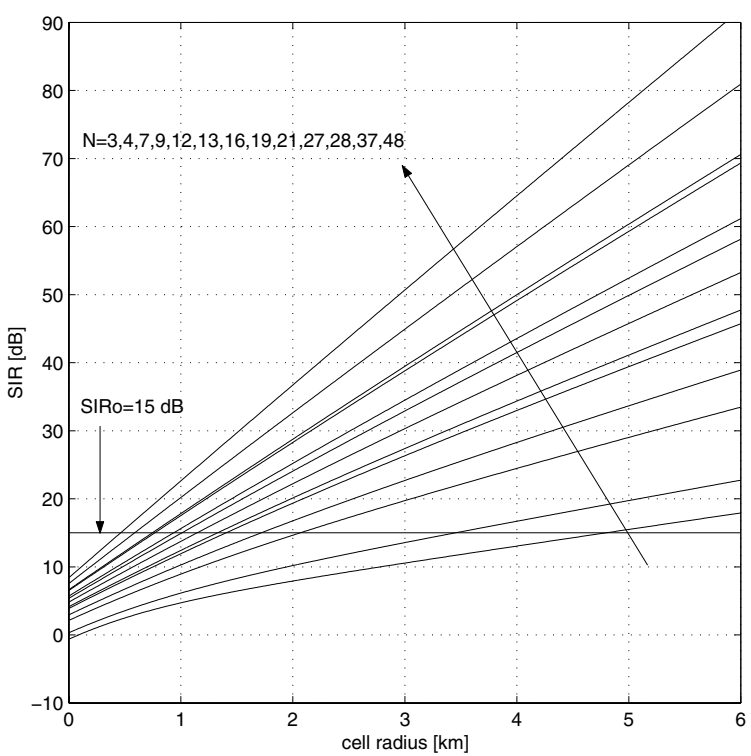

Fig. 4. SIR as a function of the cell radius $R$ for different values of the reuse number $N$.

First, let us analyze the second system constraint, namely the requirement that the per-user bandwidth be $W \geq W_{0}$. For a given density of users $\rho$, the number of users per cell is $\rho \alpha R^{2}$, where $\alpha=3 \sqrt{3} / 2$ for the hexagonal cell geometry ( $\alpha=\pi$ if the cells are modeled as circular). The bandwidth allocated to one cell is $B / N$, and, hence, the bandwidth per user must satisfy

$$
W=\frac{B / N}{\rho \alpha R^{2}} \geq W_{0}
$$

In order for this condition to hold, the cell radius has to be less than some maximum, which we express as

$$
R \leq R_{1}(N)=\frac{1}{\sqrt{\alpha \rho}} \sqrt{\frac{B}{N W_{0}}}
$$


One may also want to specify the number of users per cell as an integer, in which case the condition (10) should be re-stated to reflect the worst case,

$$
\frac{B / N}{\left\lceil\rho \alpha R^{2}\right\rceil} \geq W_{0}
$$

which would imply the maximal cell radius

$$
\underline{R}_{1}(N)=\frac{1}{\sqrt{\alpha \rho}} \sqrt{\left\lfloor\frac{B}{N W_{0}}\right\rfloor}
$$

Without the loss of generality, we will use the definition (11) in what follows.

Finally, the number of users in a cell should be greater than one, as the cellular concept is otherwise meaningless. This fact yields an additional condition,

$$
R \geq \frac{1}{\sqrt{\alpha \rho}}
$$

Combining the requirements (8), (11) and (14), we find that the cell radius must satisfy

$$
\bar{R}_{0}(N)=\max \left\{R_{0}(N), \frac{1}{\sqrt{\alpha \rho}}\right\} \leq R \leq R_{1}(N)
$$

This expression defines the admissible region of $(R, N)$. Only those values of $(R, N)$ that belong to this region constitute a valid design.

Fig.5 illustrates the admissible region for a system with $\rho=0.25$ users $/ \mathrm{km}^{2}, B=50 \mathrm{kHz}, \mathrm{SIR}_{0}=15 \mathrm{~dB}$, and $W_{0}=1 \mathrm{kHz}$. This region is bounded by $R_{0}(N), R_{1}(N)$, and the straight line $1 / \sqrt{\alpha \rho}$. Markers are placed on the curves to indicate possible values of $N(3,4,7$, etc.).

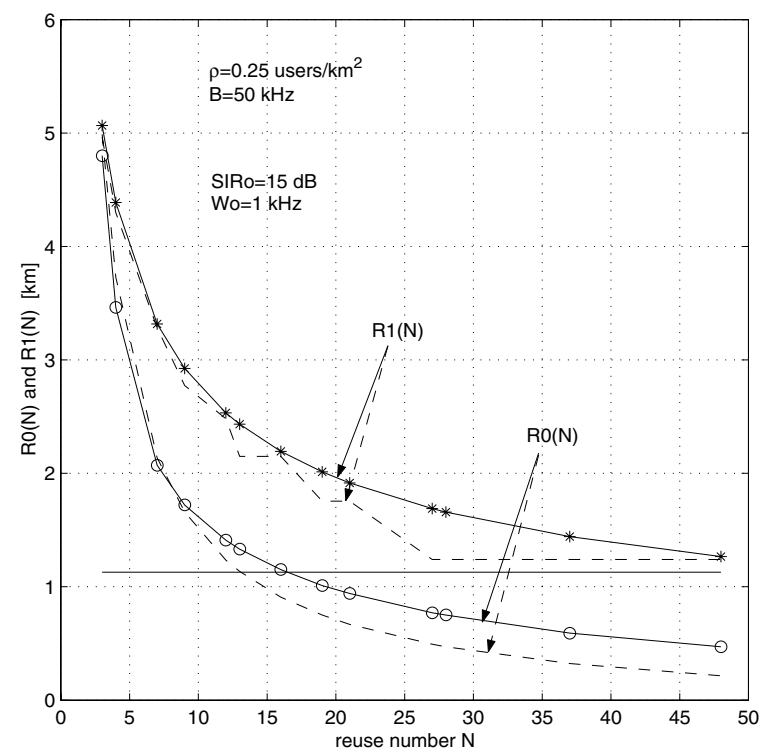

Fig. 5. Region of acceptable values $(R, N)$ is defined by (15): it bounded by $R_{0}(N), R_{1}(N)$, and the straight line which lies at $1 / \sqrt{\alpha \rho}$. Shown in dashed line are the approximation (9) for $R_{0}(N)$, and $\underline{R}_{1}(N)$ defined in (13).
We observe that $R_{0}(N)$ decays faster than $R_{1}(N)$. The smallest value of $N$ for which $R_{0}(N)$ falls below $R_{1}(N)$ defines the lowest reuse pattern that can be used. The point at which $R_{1}(N)$ falls below $1 / \sqrt{\alpha \rho}$ determines the maximal reuse number. For each admissible value of $N$, there is a range of cell radii that can be chosen to design the system. In practice, it is desirable to use a small value of $N$ because it facilitates the frequency allocation process and minimizes the loss incurred by insertion of the necessary guard bands. In this example, $N$ can be chosen as low as 3 . However, we may want to choose $N$ somewhat greater than the minimum, in order to ensure a margin for the selection of the cell radius.

\section{Number of users and bandwidth per user}

Once the reuse number is fixed, the cell radius $R$ can be chosen as any value between the minimum $\bar{R}_{0}(N)$ and the maximum $R_{1}(N)$. The selection depends on the system optimization criterion. A natural criterion to consider is maximization of the number of users supported per $\mathrm{Hz}$ of occupied bandwidth,

$$
C=\frac{\rho \alpha R^{2}}{B / N}
$$

To maximize this quantity for a given $N$, while respecting the constraints $\operatorname{SIR} \geq \operatorname{SIR}_{0}$ and $W \geq W_{0}$, the maximum value of the cell radius should be chosen, $R=R_{1}(N)$. This selection results in the maximum $C_{\max }=1 / W_{0}$, and favors SIR over the bandwidth that can be allocated to each user. Whenever the cell radius is chosen greater than $R_{0}(N)$, the SIR will be greater than the design value $\operatorname{SIR}_{0}$; when $R=R_{1}(N)$, the SIR will equal some $\operatorname{SIR}_{\text {max }}(N)$.

Alternatively, it may be desired that the per-user bandwidth $W$ be maximized. In that case, the smallest cell radius should be chosen, $R=\bar{R}_{0}(N)$. This selection yields the maximum

$$
W_{\text {max }}(N)=\frac{B / N}{\rho \alpha \bar{R}_{0}^{2}(N)}
$$

The corresponding number of users per $\mathrm{Hz}$ of occupied bandwidth is $C_{\min }(N)=1 / W_{\max }(N)$.

Hence, depending upon the chosen $N$, the actual available bandwidth per user $W$, the SIR, the number of users per $\mathrm{Hz}$ of occupied bandwidth $C$, and the associated number of users per cell $U=\rho \alpha R^{2}$, depend on the cell radius, and have a value that lies between some minimum and maximum. Fig.6 illustrates the bounds on these quantities.

\section{Sensitivity to system parameters and performance require- ments}

In the example considered, there is a large range of admissible $(R, N)$. However, a slight change in the system requirements $\left(\operatorname{SIR}_{0}, W_{0}\right)$ and/or system parameters $(\rho, B)$ may lead to a situation in which the range of solutions narrows, and also to a situation in which there is no solution. These situations are illustrated in Fig.7. 

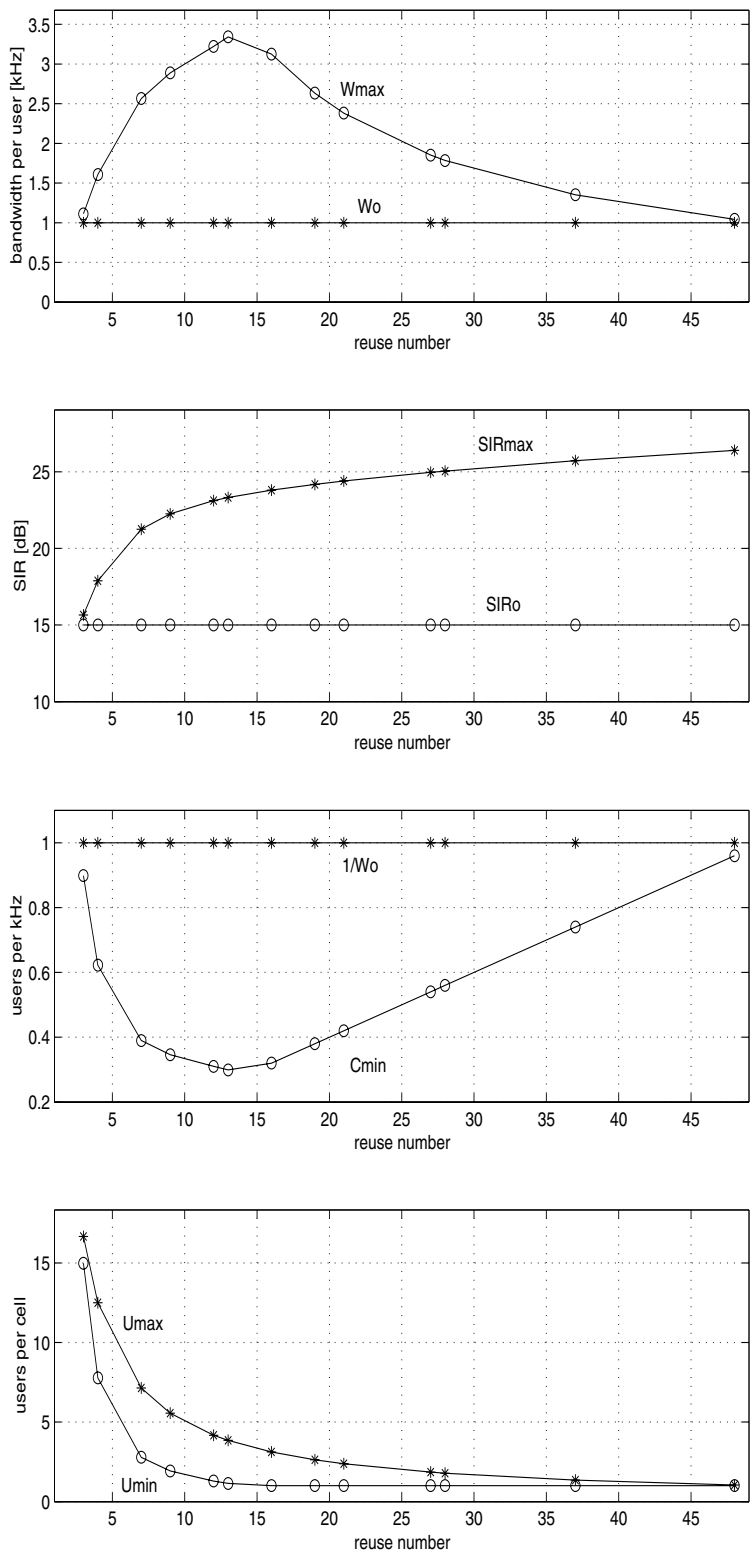

Fig. 6. Bounds on the available bandwidth per user $W$, number of users per $\mathrm{Hz}$ of occupied bandwidth $C$, SIR, and the number of users per cell $U$, all as functions of the reuse number $N$. Depending upon the cell radius, these quantities assume values between the minima and the maxima shown. Curves labeled with circles correspond to the selection of the cell radius as $R=\bar{R}_{0}(N)$, and those labeled with stars correspond to $R=R_{1}(N)$. System parameters are the same as those for Fig.5: $\rho=0.25$ users $/ \mathrm{km}^{2}, B=50 \mathrm{kHz}$, $\mathrm{SIR}_{0}=15 \mathrm{~dB}, W_{0}=1 \mathrm{kHz}$.

An increase in the required $\operatorname{SIR}_{0}$ causes $R_{0}(N)$ to increase, while an increase in the required $W_{0}$ causes $R_{1}(N)$ to decrease. Hence, increasing the requirements on the system performance $\left(\mathrm{SIR}_{0}, W_{0}\right)$ causes the region of admissible $(R, N)$ to narrow. This fact is illustrated in the upper plot, which shows the admissible region $(R, N)$ for the same system parameters $(\rho, B)$ as before, but stricter requirements $\operatorname{SIR}_{0}=17 \mathrm{~dB}$ and $W_{0}=1.5 \mathrm{kHz}$. The lower plot shows an example when there is no solution that can accommodate the required $\left(\mathrm{SIR}_{0}, W_{0}\right)$ for the specified $(\rho, B)$. In such a situation, it is not possible to employ a cellular system architecture to design the network.
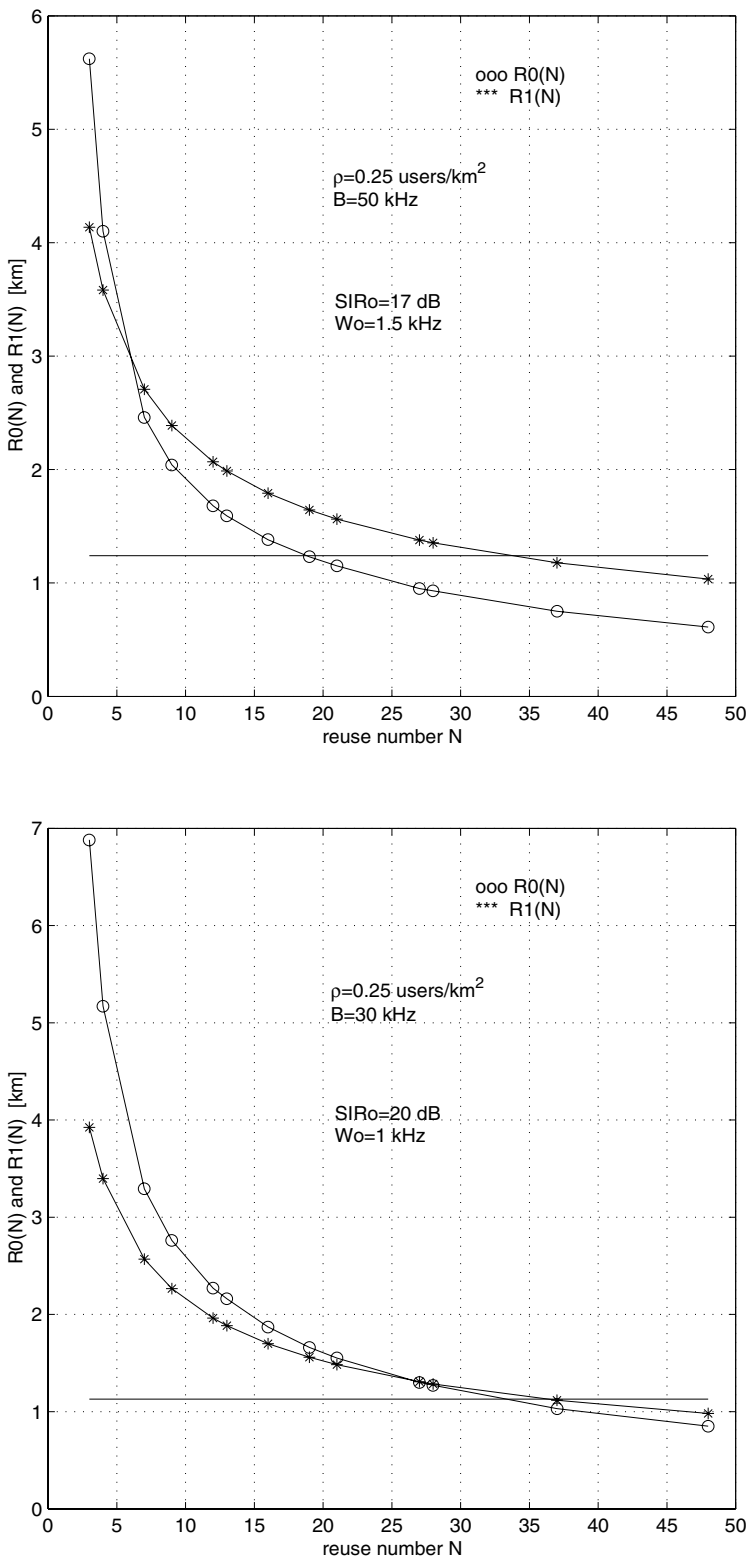

Fig. 7. Upper plot: Region of acceptable $(R, N)$ narrows for stricter system requirements $\left(\mathrm{SIR}_{0}, W_{0}\right)$ as compared to those of Fig.5. Lower plot: There are no solutions $(R, N)$ that can accommodate these system requirements $\left(\mathrm{SIR}_{0}, W_{0}\right)$ for the specified $(\rho, B)$.

Note that while $R_{0}(N)$ is governed by $\operatorname{SIR}_{0}$, and $R_{1}(N)$ by $W_{0}$ and $\rho$, both radii depend on the bandwidth $B$. We must also keep in mind that $R_{0}(N)$ depends on $f_{\text {min }}$. Fig.8 illustrates this dependence. We observe that $R_{0}(N)$ is heavily influenced by the band-edge frequency $f_{\min }$. Moving to higher frequencies improves the SIR (recall Fig.3), which in turn allows $R_{0}(N)$ to be reduced, effectively opening up the range of admissible solutions.

As an example, let us consider a system with the same requirements $\left(\mathrm{SIR}_{0}, W_{0}\right)$ as those of Fig.5, the same total bandwidth $B=50 \mathrm{kHz}$, but the user density $\rho=5$ users $/ \mathrm{km}^{2}$. Compared to $\rho=0.25$ users $/ \mathrm{km}^{2}$ (one user per $2 \mathrm{~km} \times 2 \mathrm{~km}$ ), 
this is an increase by a factor of 20 in the user density, and there is no solution with $f_{\min }=10 \mathrm{kHz}$. However, if the operational frequency range is moved to $f_{\min }=20 \mathrm{kHz}$, the range of solutions opens up, as illustrated in Fig.9. The system can now be designed with $N=7$. The price to be paid for this solution is in the increased transmission power - a higher power is needed to make up for the increased signal absorption at higher frequencies.

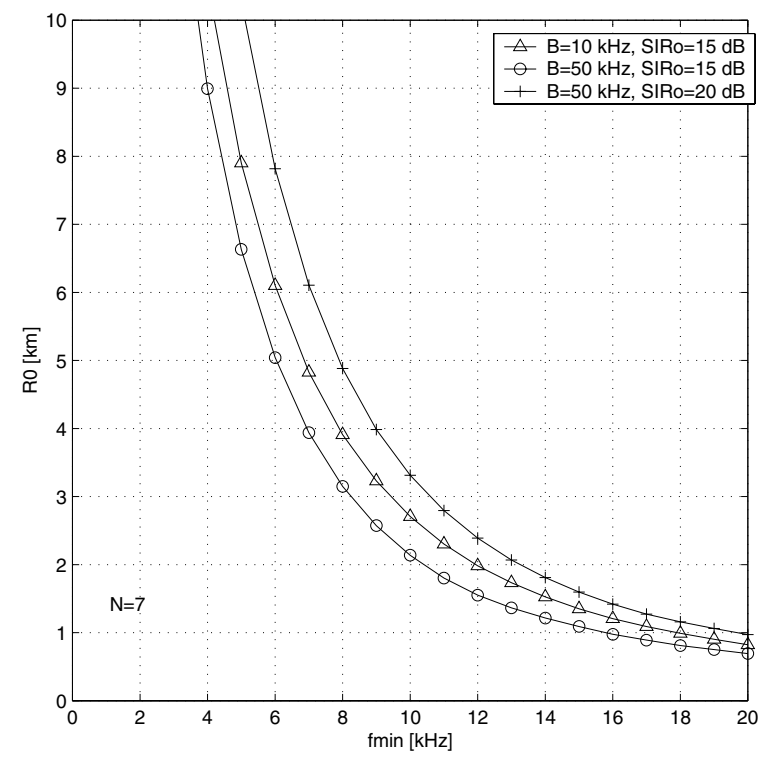

Fig. 8. $R_{0}(N)$ as a function of $f_{\min }$ for several values of $B$ and $\mathrm{SIR}_{0}$.

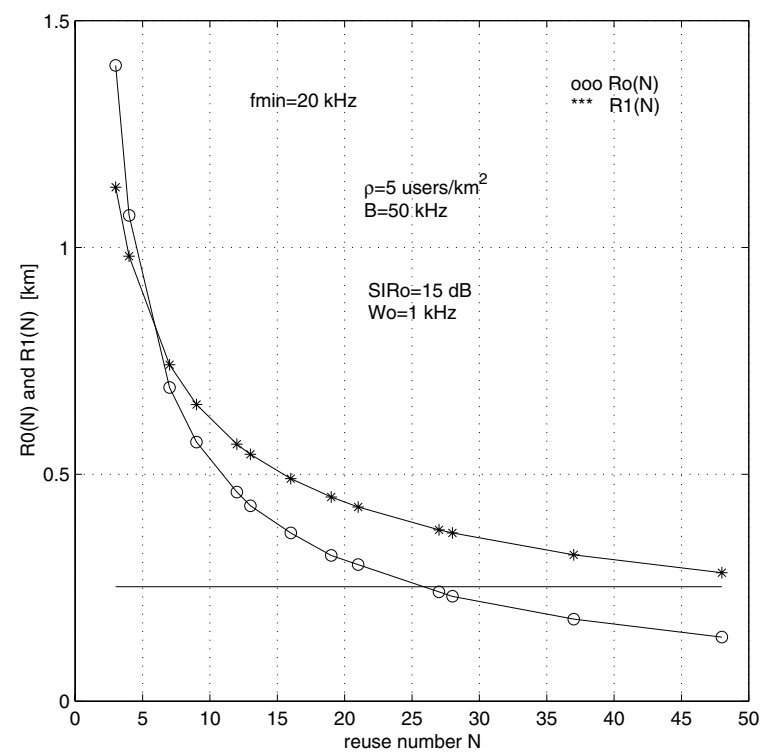

Fig. 9. Region of admissible $(R, N)$ : for the same system requirements $\left(\mathrm{SIR}_{0}, W_{0}\right)$ as those of Fig.5, a greater user density can be supported within the same bandwidth by moving the operational frequency range to higher frequencies.

\section{CONCLUSION}

Cellular frequency reuse was addressed for underwater acoustic networks where wide area coverage is required, but limited bandwidth is available. The analysis presented takes into account the physical laws of acoustic propagation, revealing interesting conclusions that emphasize the fact that existing solutions from the terrestrial radio systems do not always apply to the underwater acoustic environment.

The basic acoustic propagation loss was shown to lead to a set of constraints that the cell radius and the reuse pattern $(R, N)$ must satisfy in order to constitute an admissible solution for the cellular system topology. In particular, for the given user density and the system bandwidth $(\rho, B)$, and the quality constraints $\left(\mathrm{SIR}_{0}, W_{0}\right)$ on the signal to interference ratio and the bandwidth per user, only those values of $(R, N)$ that belong to a certain region provide a solution to the system design. The region of admissible $(R, N)$ - if existent defines the possible network topologies. This result is in stark contrast to the land-based radio systems, where $\mathrm{SIR}_{0}$ alone determines the reuse number, bearing no influence on the cell radius. Moreover, the design of an acoustic system is heavily influenced by the selection of the operational frequency range. Moving to a range of frequencies higher than that dictated by the simple SNR maximization improves the SIR, which in turn enable support of a greater number of users.

The complex relationships involved in the system design give rise to the question of system capacity, i.e. the maximal user density that can be supported within a given bandwidth. Currently, there are no analytical results that can be used to estimate this quantity. However, the analysis presented offers a framework for answering this question. Namely, the design constraints that govern the selection of the cell radius (15) also imply a relationship between the user density and the system bandwidth that must hold in order for an admissible solution to exist. These results are deferred to a later publication.

The constraints that acoustic propagation imposes on the system design, and, consequently, on its capacity, serve as a motivation for further research into the spatial reuse methods that will enable effective interference mitigation while remaining efficient in terms of both bandwidth and power usage. One such method, which capitalizes on the long propagation delay of the acoustic channel, is investigated in [4].

\section{ACKNOWLEDGMENT}

This work was supported by one or more of the following NSF grants: 0532223, 0520075, 0427502.

\section{REFERENCES}

[1] T.S.Rappaport, "Wireless Communications: Principles and Practice," Englewood Cliffs, NJ: Prentice Hall, 1996.

[2] L.Berkhovskikh and Y.Lysanov, Fundamentals of Ocean Acoustics" New York: Springer, 1982.

[3] M.Stojanovic, "On the relationship between capacity and distance in an underwater acoustic channel," in Proc. First ACM International Workshop on Underwater Networks (WUWNet), Sept. 2006.

[4] B.Peleato and M.Stojanovic, "A Channel sharing scheme for underwater cellular networks," in Proc. IEEE Oceans Europe Conf., 2007. 